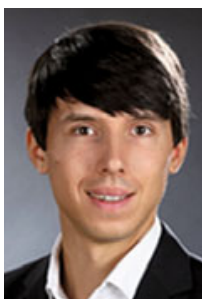

Sascha Hein

Jahrgang 1991. 2010-2015 Studium Biomolecular Engineering an der TU Darmstadt, Schwerpunkt: Weiße Biotechnologie; dort 2015-2019 Promotion am Fachbereich Biologie. Seit 2019 Postdoc in der Gruppe von Prof. Dr. J. Simon, TU Darmstadt.

DOI: $10.1007 / \mathrm{s} 12268-020-1389-\mathrm{Z}$ (C) Der Autor 2020

Menachinone sind aufgrund ihrer hydrophoben Eigenschaften membranständige Moleküle und kommen in sehr vielen Mikroorganismen vor. Sie lassen sich mittels zwei Elektronen zur jeweiligen Menachinol-Form reduzieren. In der Membran von Bakterien und Archaeen bilden Chinone einen Chinonpool und fungieren als universelle Redoxmediatoren. Mikroorganismen aus anaeroben Habitaten besitzen im Chinonpool oft neben dem klassischen Menachinon (MK) methylierte MK-Derivate wie Methylmenachinon (MMK) und Dimethylmenachinon (DMMK). Da die methylierten Menachinone bei negativeren Standardredoxpotenzialen als MK operieren, spielen sie eine entscheidende Rolle für spezifische Elektronentransportketten der anaeroben Atmung.

Das Ziel meiner Doktorarbeit war es, die Biosynthese von methylierten Menachinonen und die Position der zusätzlichen Methylgruppen zu entschlüsseln. Als Modellorganismen dienten die MMK-produzierenden Bakterien Wolinella succinogenes und Adlercreutzia equolifaciens. Stromaufwärts des Polysulfidreduktase-Genclusters von $W$. succinogenes identifizierten wir das Gen für eine radikalische $S$-Adenosylmethionin-Methyltransferase (RSMT) der Klasse C [1]. Mithilfe von entsprechenden Deletions- und Komplementationsmutanten stellten wir fest, dass diese Methyltransferase für die MMKSynthese benötigt wird (Abb. 1A, [1]). Homolo-

VAAM-Promotionspreis 2020

\title{
Effizienter atmen - Biosynthese methylierter Menachinone
}

\author{
SASCHA HEIN \\ MIKROBIELLE ENERGIEUMWANDLUNG UND BIOTECHNOLOGIE, TU DARMSTADT
}

ge dieser Methyltransferase identifizierten wir in diversen MMK- und DMMK-produzierenden Mikroorganismen und nannten sie abhängig vom jeweiligen MK-Biosyntheseweg MenK oder MqnK. Nach heterologer Expression der mqnK/ menK-Gene aus $W$. succinogenes, A. equolifaciens oder Shewanella oneidensis in Escherichia coli bildet sich nicht nur das methylierte Menachinon, sondern auch das bisher nicht beschriebene Methyl-2-demethylmenachinon. Zur Bestimmung der exakten Position der Methylgruppe wurden die Menachinone mittels Hochleistungsflüssigkeitschromatographie (HPLC) aus den Organismen gereinigt und mit hochauflösender Massenspektrometrie und zweidimensionaler NMR untersucht [1]. Im HMBC (heteronuclear multiple bond correlation)-NMR-Spektrum zeigte sich die methylierte Position als das Kohlenstoffatom C-8 (Abb. 1B).

In DMMK-produzierenden Organismen gelang die Identifizierung eines weiteren MenK-Homologs: MenK2 [2]. Es stellte sich heraus, dass dieses Enzym die Position C-7 von MK methyliert (Abb. 1A). Dies bedeutet, dass während der Biosynthese von DMMK die Methylgruppen von zwei unterschiedlichen Enzymen auf Menachinon übertragen werden (Abb. 1B).

Zum besseren funktionalen Verständnis dieser Enzymklasse erfolgte die heterologe Produktion von MenK aus A. equolifaciens in $E$. coli mit anschließender Reinigung. Es zeigte sich, dass MenK, wie alle RSMTs, ein sauerstoffsensibles und redoxaktives [4Fe-4S]-Zentrum

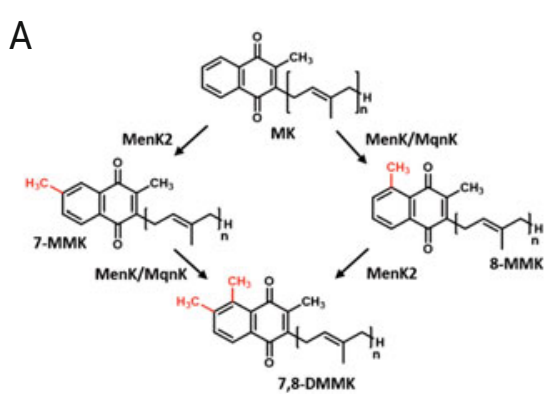

besitzt. In einem eigens entwickelten anaeroben Enzymtest bestimmte ich im Anschluss mittels LC-MS die Produktstöchiometrie und zeigte außerdem mit deuterierten Substraten, dass die Methylierung des nicht nukleophilen Kohlenstoffatoms über einen radikalischen Mechanismus erfolgt.

Mithilfe dieser Ergebnisse ist es möglich, mqnK/menK/menK2-Gene als Biomarker zur Vorhersage der MMK- und DMMK-Produktion zu nutzen. In Zukunft kann dieses Wissen essenziell sein, um biotechnologische Prozesse zur Methylierung von inerten $s p^{2}$-hybridisierten Kohlenstoffatomen zu entwickeln.

\section{Danksagung}

Mein größter Dank gilt meinem Betreuer Jörg Simon und seiner Arbeitsgruppe für die tägliche Unterstützung. Des Weiteren möchte ich Reinhard Meusinger, Markus Gallei und Heribert Warzecha für die tolle Kooperation danken.

\section{Literatur}

[1] Hein S, Klimmek O, Polly M et al. (2017) A class C radical $S$-adenosylmethionine methyltransferase synthesizes 8-methylmenaquinone. Mol Microbiol 104:449-462 [2] Hein S, von Irmer J, Gallei M et al. (2018) Two dedicated class C radical $S$-adenosylmethionine methyltransferases concertedly catalyse the synthesis of 7,8-dimethylmenaquinone. Biochim Biophys Acta Bioenerg 1859:300-308

Funding: Open Access funding provided by Projekt DEAL.

Open Access: Dieser Artikel wird unter der Creative Commons Namensnennung 4.0 International Lizenz veröffentlicht, welche die Nutzung, Vervielfältigung, Bearbeitung, Verbreitung und Wiedergabe in jeglichem Medium und Format erlaubt, sofern Sie den/die ursprünglichen Autor(en) und die Quelle ordnungsgemäß nennen, einen Link zur Creative Commons Lizenz beifügen un angeben, ob Änderungen vorgenommen wurden. Die in diesem Artikel enthaltenen Bilder und sonstiges Drittmaterial unterliegen ebenfalls der genannten Creative Commons Lizenz, sofern sich aus der Abbildungslegend nichts anderes erght. Sorm das bets tente Material nicht unter der gach Wechteinhabers einzuholen. Weitere Details zur Lizenz entnehmen Sie bitte der Lizenzinformation auf http://creativecommons.org/licenses/by/4.0/deed.de.

Korrespondenzadresse:

Dr. Sascha Hein

AG Simon

Technische Universität Darmstadt

Schnittspahnstraße 10

D-64287 Darmstadt

hein@bio.tu-darmstadt.de

Abb. 1: Biosynthese und Struktur methylierter Menachinon-Derivate. A, Biosynthese von Methylmenachinon (MMK) und Dimethylmenachinon (DMMK) ausgehend von Menachinon (MK). Die Spezifität der identifizierten Enzyme bestimmt die Position der Methylierung. So methyliert MenK/MqnK stets an Position C-8 und MenK2 an Position C-7. Die Biosynthese von DMMK findet in zwei Stufen statt. B, Ausschnitt des HMBC-NMR-Spektrums von 8-Methylmenachinon (8-MMK). Mittels ${ }^{4}$ J-Kopplung zwischen $\mathrm{C}-1$ und den Protonen an $\mathrm{C}-13$ gelang die Bestimmung der Position der Methylgruppe an C-8.
Die VAAM dankt den Sponsoren der Promotionspreise: BASF SE, Bayer AG, New England Biolabs GmbH und Evonik Industries AG. 